\title{
Investigation of patients with coronary slow flow in terms of periodontal health status
}

\author{
Emrah Erdal' (1)*, Gülbahar Ustaoğlu² (D), Zeynep Karaș² (D), Satılmıș Bilgin³
}

\section{SUMMARY}

OBJECTIVE: This study aims to evaluate the relationship between periodontal health status and coronary slow flow phenomenon. METHODS: One hundred and two patients who underwent coronary angiography with the diagnosis of stable angina pectoris were included in the study. Patients were divided into two groups: patients with coronary slow flow (Test group) ( $n=51$ ), and patients with normal coronary angiography (Control group) $(n=51)$. Diagnosis of slow coronary flow was made according to Beltrame criteria by coronary angiography. Demographic characteristics of the participants were recorded. The periodontal health was assessed by clinical periodontal parameters such as probing depth, clinical attachment level, gingival index, plaque index, and bleeding on probing.

RESULTS: There were no significant differences between groups as regards the frequencies of hypertension, smoking ( $p>0.05)$. As for the periodontal parameters of the study groups, probing depth, gingival index, plaque index, bleeding on probing, and clinical attachment level values were statistically higher in the test group compared to the control group $(p<0.05)$.

CONCLUSIONS: Periodontitis might be accepted as one of the underlying causes of coronary slow flow. Patients with coronary slow flow should be evaluated for an underlying periodontal disease, and treatment of periodontal disease can protect against future cardiovascular events.

KEYWORDS: Periodontal diseases. Angiography. Slow-flow phenomenon. Coronary artery disease.

\section{INTRODUCTION}

The coronary slow flow (CSF) phenomenon first introduced in 1972 by Tambe et al. in six patients with chest pain, is the late removal of opaque material from normal and/or near-normal epicardial coronary vessels during coronary angiography'. The prevalence in patients undergoing coronary angiography for stable angina pectoris is about $1-5 \%^{2}$. CSF is associated with several clinical cases such as arrhythmia, angina pectoris, acute myocardial infarction (MI), and sudden death ${ }^{3}$.
Information about the etiology of CSF is insufficient. microvascular endothelial dysfunction, Inflammation, and increased coronary microvascular resistance are recommended as the underlying physiopathological causes ${ }^{4}$.

Periodontitis is a multifactorial chronic inflammatory disease caused by dysbiotic plaque biofilms and described as progressive destruction of supportive tissues of teeth ${ }^{5}$. It does not affect only the oral cavity and influences general health. It may induce endotoxemia, bacteremia, and

\footnotetext{
'Bolu Abant Izzet Baysal University, Medical Faculty, Department of Cardiology - Bolu, Turkey.

${ }^{2}$ Bolu Abant Izzet Baysal University, Dentistry Faculty, Department of Periodontology - Bolu, Turkey.

${ }^{3}$ Bolu Abant Izzet Baysal University, Medical Faculty, Department of Internal Medicine - Bolu, Turkey.

*Corresponding author: dr.emraherdal@gmail.com

Conflicts of interest: the authors declare there are no conflicts of interest. Funding: none.

Received on January 03, 2021. Accepted on January 09, 2021.
} 
systemic low-grade inflammation. Furthermore, recent studies reported that periodontitis is commonly seen in patients with cardiovascular disease ${ }^{6,7}$.

Although the role of periodontitis as an independent risk factor for atherosclerotic CVDs is established, there is no study evaluating a possible relationship between periodontitis and CSF. In the present study, we aimed to assess the association between periodontal health and coronary slow flow phenomenon.

\section{METHODS}

\section{Study population}

This is a prospective study that was conducted in the School of Medicine of Bolu Abant Izzet Baysal University between June 2019 and February 2020. The study has been conducted following the principles of the Declaration of Helsinki and approved by the local Institutional Review Board. Written informed consent was obtained from all subjects.

Type I errors (0.05), targeted power (0.80), and effect size $(0.50)$ due to probing depth $(\mathrm{PD})$ value $(\mathrm{p}<0.05)$ by $\mathrm{G}^{*}$ power 3.1.9.4 software program (Heinrich Heine University, Dusseldorf, Germany) were considered for the calculation of the sample size ${ }^{8}$. The minimum required sample size was calculated as 51 .

A total of 102 patients who experienced coronary angiography with the diagnosis of stable angina pectoris were enrolled for the study. While patients with coronary slow flow were determined as the test group (group 1, $\mathrm{n}=51$ ), patients with normal coronary angiography were assigned as a control group (group 2, $\mathrm{n}=51$ ). Demographic characteristics of the participants were recorded. The periodontal health of subjects was also evaluated by clinical periodontal parameters.

Diagnosis of slow coronary flow was made according to Beltrame criteria; Angiographic evidence of CSFP, described by: a) No evidence of obstructive epicardial coronary artery disease (CAD) (no lesions $\geq 40 \%$ ), b) Delayed distal vessel contrast opacification as evidenced by either: TIMI 2 flow (requiring $\geq 3$ beats to opacify the vessel) or corrected TIMI frame count $>27$ frames (images acquired at 30 frames/s), c) Delayed distal opacification in at least 1 epicardial vessel?.

Individuals with the following conditions were excluded: patients who had previously undergone percutaneous intervention or bypass surgery, had periodontal treatment within 6 months, are using antiarrhythmic drugs, had coronary ectasia, diabetes mellitus, systemic diseases predisposing to periodontal diseases, including immune deficiency and autoimmunity; used antibiotics and/or anti-inflammatory drugs in the last 6 months.

\section{Periodontal examination}

All clinical periodontal parameters were evaluated by the same periodontist (G.U), and a calibration exercise was done to obtain acceptable interexaminer reproducibility. Periodontal examinations were performed with a Williams probe (Hu-Friedy, Chicago, IL, USA). The clinical parameters of $\mathrm{PD}$, clinical attachment level (CAL) gingival index $(\mathrm{GI})^{10}$, and plaque index $(\mathrm{PI})^{11}$ were measured for every tooth present in the oral cavity. The measurements were performed at six sites (mesiobuccal, midbuccal, distobuccal, mesiolingual, midlingual, distolingual) and the results were recorded in approximation to the nearest whole millimeter. The distance from the bottom of the pocket to the cementoenamel junction was defined as CAL and this distance between these two points was measured and recorded (Figure 1). The mean PD and the mean CAL values were calculated by dividing the total score of all teeth by the total number of teeth examined during the study. The periodontal probe was carefully and gently introduced into the gingival sulcus to calculate the percentage of $\mathrm{BOP}$, even one site with BOP was recorded as (+) for each tooth.

\section{Loe \& silness gingival index ${ }^{10}$}

Score 0: Normal gingiva; Score 1: Slight inflammation - slight change in color, slight edema. No bleeding on probing; Score 2: Moderate inflammation - redness, edema, glazing. Bleeding on



Figure 1. Measurement of clinical attachment level with a Williams Probe. 
probing; Score 3: Severe inflammation - noticeable redness and edema, ulceration. A tendency toward spontaneous bleeding.

\section{Silness \& loe piaque index ${ }^{11}$}

Score 0: Absence of microbial plaque; Score 1: Thin film of microbial plaque along the free gingival margin; Score 2: Moderate accumulation with plaque in the sulcus; Score 3: A large amount of plaque in sulcus or pocket along the free gingival margin.

\section{Statistical analysis}

Data were analyzed using the IBM Statistical Package for Social Sciences v15 (SPSS Inc., Chicago, IL, USA). Data are expressed as mean $\pm \mathrm{SD}$ or median (interquartile range), as appropriate. To evaluate the differences between the two groups, The Student t-test was used for normally distributed variables and Mann-Whitney's U-test was performed for non-parametric variables. The Chi-square test was used for qualitative variables. All differences associated with a chance probability of .05 or less were considered statistically significant.

\section{RESULTS}

The median ages were 50.5 (11) and 47 (8.7) years for the test and control group, respectively. No significant difference was observed for age and gender between the study groups. There were no significant differences between groups as regards the frequencies of, hypertension, smoking ( $p>0.05$ ) (Table 1).

As for the periodontal parameters of the study groups, PD, GI, PI, BOP, and CAL values were statistically higher in the test group compared to the control group $(\mathrm{p}<0.05)$ (Table 2).

\section{DISCUSSION}

Although the studies attributed the etiology of CSF to microvascular and endothelial dysfunction, widespread atherosclerosis, inflammation, and platelet dysfunction, its pathophysiology has not been clarified. We aimed to evaluate the periodontal health status of CSF patients and to detect underlying periodontal disease.

Table 1. General characteristics of the study groups.

\begin{tabular}{l|c|c|c} 
Baseline characteristics & $\begin{array}{c}\text { Group 1 }(\mathrm{n}=51) \\
\text { Test group }\end{array}$ & $\begin{array}{c}\text { Group 2 (n=51) } \\
\text { Control group }\end{array}$ & p-value \\
\hline Age [median (IQR)] & $50.5(11)$ & $47(8.7)$ & 0.080 \\
\hline HT [number (\%)] & $6(13.3)$ & $10(19.5)$ & 0.384 \\
\hline Sex (female/male) & $18 / 33$ & $21 / 30$ & 0.627 \\
\hline Smoking [number (\%)] & $6(11.7)$ & $9(17.6)$ & 0.337 \\
\hline Number of slow flow coronary arteries (\%) & $27(60)$ & - & \\
\hline One vessel & $9(20)$ & - & \\
\hline Two vessels & $9(20)$ & - & \\
\hline Tree vessels & &
\end{tabular}

HT: hypertension; IQR: interquartile range.

Table 2. Comparison of periodontal parameters of the study groups.

\begin{tabular}{l|c|c|c} 
& $\begin{array}{c}\text { Group 1 }(\mathrm{n}=51) \\
\text { Test group } \\
\text { median (IQR) }\end{array}$ & $\begin{array}{c}\text { Group 2 }(\mathrm{n}=51) \\
\text { Control group } \\
\text { median (IQR) }\end{array}$ & p-value \\
\hline $\mathrm{PD}$ & $2.28(0.8)$ & $1.88(0.4)$ & $<0.001$ \\
\hline $\mathrm{CAL}$ & $2.27(1.0)$ & $2.0(0.4)$ & 0.001 \\
\hline $\mathrm{GI}$ & $2.0(0.6)$ & $1.5(0.5)$ & $<0.001$ \\
\hline $\mathrm{PI}$ & $2.0(0.5)$ & $1.45(1.0)$ & $<0.001$ \\
\hline BOP $(\%)$ & $100(59)$ & $47(9)$ & $<0.001$ \\
\hline
\end{tabular}

PD: probing depth; CAL: clinical attachment level; Gl: gingival index; PI: plaque index; BOP: bleeding on probing. 
Periodontitis is a chronic multifactorial inflammatory disease and the systematic inflammatory response results in endothelial dysfunction, thus contributing to cardiovascular diseases ${ }^{12}$.

In our study, we found PD, CAL, GI, PI, and BOP values that are periodontal disease markers statistically higher in the test group. Therefore, periodontitis may be one of the underlying causes of this disease. Indeed, previous papers revealed a link between inflammation and CSF too.

In a study, Xia and colleagues ${ }^{13}$ found that hs-CRP levels were the most important predictor of coronary slow flow and suggested that this finding played an important role in inflammation in CSF. Likewise, Li et al. ${ }^{14}$ argued that increased inflammatory markers might be an indicator of endothelial activation and inflammation in patients with CSF. Similarly in another study, Madak et al. ${ }^{15}$ stated that the blood levels of Hs-CRP and $\mathrm{N}$-terminal pro-B-type natriuretic peptide were higher in CSF patients than the control group, and suggested that inflammation was the main factor of many cardiovascular events, and was associated with different clinical coronary artery diseases.

Recent studies have shown a strong relationship between periodontitis and systemic diseases including cardiovascular diseases and diabetes ${ }^{16,17}$. Periodontal disease was previously associated with recurrent cardiovascular events in patients with a recent $\mathrm{MI}^{6,18}$. Likewise, in the study conducted by Gürkan et al. ${ }^{8}, 28$ healthy individuals were compared with 32 isolated coronary artery disease patients. Patients with isolated coronary artery ectasia had significantly higher clinical periodontal parameters.

According to our results, the presence of periodontitis was significantly higher in the test group. In the English literature, periodontal treatment generally leads to an improvement in endothelial functions ${ }^{19-21}$. In a study, Li et al. ${ }^{22}$ reported very low-quality evidence to support whether periodontal treatment can prevent the relapse of CVD in the long term in patients with periodontitis, but no significant indication on primary prevention was found. In a recent study, Lobo et al..$^{23}$ concluded that treatment of periodontal disease improved endothelial functions in patients with MI. Similarly, Teeuw et al. ${ }^{24}$ reported that in patients with periodontitis and other comorbidities (CVD and metabolic syndrome), reductions in biomarkers of atherosclerotic disease such as CRP, interleukin -6, total cholesterol, triglyceride, high-density lipoprotein, and also improvements in endothelial functions were detected after periodontal treatment.

Similarly, periodontal treatment has been shown to result in improved metabolic control in periodontitis patients with diabetes, as demonstrated by a significant decrease in HbAlc levels in a systematic review ${ }^{25}$.

Limitations of our study; the present case-control study has a small population size. Correlation with inflammatory markers was not performed in patients with CSF. These inflammatory markers or cardiovascular events were not assessed after periodontal disease therapy.

\section{CONCLUSIONS}

Patients with CSF should be evaluated for underlying periodontal disease and treatment of periodontal disease can protect against future cardiovascular events. In addition, if we look back, patients with periodontitis should be carefully examined by dentists and well evaluated for cardiovascular diseases and other risk factors.

\section{ACKNOWLEDGMENTS}

This study was approved by the Clinical Research Ethics Committee of the XX University. All the procedures in this study were following the 1975 Declaration of Helsinki, updated in 2013.

\section{AUTHORS" CONTRIBUTIONS}

EE: Conceptualization, Project Administration, Resources, Supervision, Writing - Original Draft. GU: Conceptualization, Project Administration, Resources, Writing - Original Draft. ZK: Resources, Writing - Original Draft. SB: Supervision, Writing - Original Draft.

\section{REFERENCES}

1. Tambe AA, Demany MA, Zimmerman HA, Mascarenhas E. Angina pectoris and slow flow velocity of dye in coronary arteries--a new angiographic finding. Am Heart J. 1972;84(1):66-71. https://doi.org/10.1016/0002-8703(72)90307-9
2. Goel PK, Gupta SK, Agarwal A, Kapoor A. Slow coronary flow: a distinct angiographic subgroup in syndrome X. Angiology. 2001;52(8):507-14. https://doi. org/10.1177/000331970105200801 
3. Tatli E, Yildirim T, Aktoz M. Does coronary slow flow phenomenon lead to myocardial ischemia? Int J Cardiol. 2009;131(3):e101-2. https://doi.org/10.1016/j.ijcard.2007.07.069

4. Rosano GM, Peters NS, Kaski JC, Mavrogeni SI, Collins P, Underwood RS, et al. Abnormal uptake and washout of thallium-201 in patients with syndrome $X$ and normalappearing scans. Am J Cardiol. 1995;75(5):400-2. https:// doi.org/10.1016/s0002-9149(99)80565-7

5. Papapanou PN, Sanz M, Buduneli N, Dietrich T, Feres M, Fine $\mathrm{DH}$, et al. Periodontitis: consensus report of workgroup 2 of the 2017 World Workshop on the classification of periodontal and peri-implant diseases and conditions. J Periodontol. 2018;89(Suppl 1):S173-82. https://doi.org/10.1002/JPER.170721

6. Rydén L, Buhlin K, Ekstrand E, de Faire U, Gustafsson A, Holmer J, et al. Periodontitis Increases the Risk of a First Myocardial Infarction: A Report From the PAROKRANK Study. Circulation. 2016;133(6):576-83. https://doi.org/10.1161/ CIRCULATIONAHA. 115.020324

7. Nordendahl E, Gustafsson A, Norhammar A, Näsman P, Rydén L, Kjellström B. Severe periodontitis is associated with myocardial infarction in females. J Dent Res. 2018;97(10):1114-21. https:// doi.org/10.1177/0022034518765735

8. Gürkan U, Yağmur S, Akgöz H, Aksoy S, Oz D, Akyüz S, et al. Severity of periodontitis in patients with isolated coronary artery ectasia. Int Heart J. 2014;55(4):296-300. https://doi. org/10.1536/ihj.13-361

9. Beltrame JF. Defining the coronary slow flow phenomenon. Circ J. 2012;76(4):818-20. https://doi.org/10.1253/circj. cj-12-0205

10. Löe $H$. The gingival index, the plaque index and the retention index systems. J Periodontol. 1967;38(6):Suppl:610-6. https:// doi.org/10.1902/jop.1967.38.6.610

11. Silness J, Loe H. Periodontal disease in pregnancy. li. Correlation between oral hygiene and periodontal condtion. Acta Odontol Scand. 1964;22:121-35. https:// doi.org/10.3109/00016356408993968

12. Packard RR, Libby P. Inflammation in atherosclerosis: from vascular biology to biomarker discovery and risk prediction. Clin Chem. 2008;54(1):24-38. https://doi.org/10.1373/ clinchem.2007.097360

13. Xia S, Deng SB, Wang Y, Xiao J, Du JL, Zhang Y, et al. Clinical analysis of the risk factors of slow coronary flow. Heart Vessels. 2011;26(5):480-6. https://doi.org/10.1007/ s00380-010-0081-5

14. Li JJ, Qin XW, Li ZC, Zeng HS, Gao Z, Xu B, et al. Increased plasma C-reactive protein and interleukin- 6 concentrations in patients with slow coronary flow. Clin Chim Acta. 2007;385(1-2):43-7. https://doi.org/10.1016/j. cca.2007.05.024
15. Madak N, Nazlı Y, Mergen H, Aysel S, Kandaz M, Yanık E, et al. Acute phase reactants in patients with coronary slow flow phenomenon. Anadolu Kardiyol Derg. 2010;10(5):41620. https://doi.org/10.5152/akd.2010.139

16. Arana C, Moreno-Fernández AM, Gómez-Moreno G, MoralesPortillo C, Serrano-Olmedo I, de la Cuesta Mayor MC, et al. Increased salivary oxidative stress parameters in patients with type 2 diabetes: Relation with periodontal disease. Endocrinol Diabetes Nutr. 2017;64(5):258-64. https://doi.org/10.1016/j. endinu.2017.03.005

17. Corlan Pușcu D, Ciuluvică RC, Anghel A, Mălăescu GD, Ciursaș AN, Popa GV, et al. Periodontal disease in diabetic patients - clinical and histopathological aspects. Rom J Morphol Embryol. 2016;57(4):1323-29. PMID: 28174799

18. Dorn JM, Genco RJ, Grossi SG, Falkner KL, Hovey KM, lacoviello $L$, et al. Periodontal disease and recurrent cardiovascular events in survivors of myocardial infarction (MI): the Western New York Acute MI Study. J Periodontol. 2010;81(4):502-11. https://doi.org/10.1902/jop.2009.090499

19. Ramírez JH, Arce RM, Contreras A. Periodontal treatment effects on endothelial function and cardiovascular disease biomarkers in subjects with chronic periodontitis: protocol for a randomized clinical trial. Trials. 2011;12:46. https://doi. org/10.1186/1745-6215-12-46

20. Seinost G, Wimmer G, Skerget M, Thaller E, Brodmann M, Gasser R, et al. Periodontal treatment improves endothelial dysfunction in patients with severe periodontitis. Am Heart J. 2005;149(6):1050-4. https://doi.org/10.1016/j.ahj.2004.09.059

21. Piconi S, Trabattoni D, Luraghi C, Perilli E, Borelli M, Pacei M, et al. Treatment of periodontal disease results in improvements in endothelial dysfunction and reduction of the carotid intimamedia thickness. FASEB J. 2009;23(4):1196-204. https://doi. org/10.1096/fj.08-119578

22. Li C, Lv Z, Shi Z, Zhu Y, Wu Y, Li L, et al. Periodontal therapy for the management of cardiovascular disease in patients with chronic periodontitis. Cochrane Database Syst Rev. 2017;11(11):Cd009197. https://doi.org/10.1002/14651858. CD009197.pub2

23. Lobo MG, Schmidt MM, Lopes RD, Dipp T, Feijó IP, Schmidt KES, et al. Treating periodontal disease in patients with myocardial infarction: a randomized clinical trial. Eur J Intern Med. 2020;71:76-80. https://doi.org/10.1016/j.ejim.2019.08.012

24. Teeuw WJ, Slot DE, Susanto H, Gerdes VE, Abbas F, D'Aiuto F, et al. Treatment of periodontitis improves the atherosclerotic profile: a systematic review and meta-analysis. J Clin Periodontol. 2014;41(1):70-9. https://doi.org/10.1111/jcpe.12171

25. Teeuw WJ, Gerdes VE, Loos BG. Effect of periodontal treatment on glycemic control of diabetic patients: a systematic review and meta-analysis. Diabetes Care. 2010;33(2):421-7. https:// doi.org/10.2337/dc09-1378 